\title{
An Improved FPT Algorithm and a Quadratic Kernel for Pathwidth One Vertex Deletion
}

\author{
Marek Cygan • Marcin Pilipczuk • \\ Michał Pilipczuk • Jakub Onufry Wojtaszczyk
}

Received: 27 December 2010 / Accepted: 16 September 2011 / Published online: 29 September 2011

(C) The Author(s) 2011. This article is published with open access at Springerlink.com

\begin{abstract}
The PAthwidth One Vertex Deletion (POVD) problem asks whether, given an undirected graph $G$ and an integer $k$, one can delete at most $k$ vertices from $G$ so that the remaining graph has pathwidth at most 1 . The question can be considered as a natural variation of the extensively studied FEEDBACK VERTEX SET (FVS) problem, where the deletion of at most $k$ vertices has to result in the remaining graph having treewidth at most 1 (i.e., being a forest). Recently Philip et al. (WG, Lecture Notes in Computer Science, vol. 6410, pp. 196-207, 2010) initiated the study of the parameterized complexity of POVD, showing a quartic kernel and an algorithm which runs in time $7^{k} n^{O(1)}$. In this article we improve these results by showing a quadratic kernel and an algorithm with time complexity $4.65^{k} n^{O(1)}$, thus obtaining almost tight kernelization bounds when compared to the general result of Dell and van Melkebeek (STOC, pp. 251-260, ACM, New York, 2010). Techniques used in the kernelization are based on the quadratic kernel for FVS, due to Thomassé (ACM Trans. Algorithms 6(2), 2010).
\end{abstract}

Keywords Fixed parameter tractability $\cdot$ Kernelization · Pathwidth · Caterpillar graph

M. Cygan · M. Pilipczuk · M. Pilipczuk $(\bowtie) \cdot$ J.O. Wojtaszczyk

Faculty of Mathematics, Computer Science and Mechanics, University of Warsaw, ul. Banacha 2, 02-097 Warsaw, Poland

e-mail: michal.pilipczuk@students.mimuw.edu.pl

M. Cygan

e-mail: cygan@mimuw.edu.pl

M. Pilipczuk

e-mail:malcin@mimuw.edu.pl

J.O. Wojtaszczyk

e-mail: onufry@mimuw.edu.pl 


\section{Introduction}

In the parameterized complexity setting, an input instance comes with an integer parameter $k$-formally, a parameterized problem $Q$ is a subset of $\Sigma^{*} \times \mathbb{N}$ for some finite alphabet $\Sigma$. We say that the problem is fixed parameter tractable $(F P T)$ if there exists an algorithm which solves any instance $(x, k)$ in time $f(k)$ poly $(|x|)$ for some (usually exponential) computable function $f$. It is known that a decidable problem is FPT iff it is kernelizable: a kernelization algorithm for a problem $Q$ is an algorithm that takes an instance $(x, k)$ and in time polynomial in $|x|+k$ produces an equivalent instance $\left(x^{\prime}, k^{\prime}\right)$ (i.e., $(x, k) \in Q$ iff $\left.\left(x^{\prime}, k^{\prime}\right) \in Q\right)$ such that $\left|x^{\prime}\right|+k^{\prime} \leq g(k)$ for some computable function $g$. The function $g$ is the size of the kernel and if it is polynomial, we say that $Q$ admits a polynomial kernel. Kernelization techniques can be viewed as polynomial time preprocessing routines for tackling NP-hard problems. Parameterized complexity provides a formal framework for the analysis of such algorithms $[8,11,15]$.

The notions of pathwidth and treewidth, introduced by Robertson and Seymour $[19,20]$, measure how much a given graph resembles a path and a tree, respectively. Both play an important role in the proof of the Graph Minor Theorem, and there exists a large family of fixed parameter algorithms (usually based on the dynamic programming principle) for problems parameterized by treewidth.

One of the most extensively studied problems in the parameterized complexity community, FEEDBACK VERTEX SET, asks for a set of vertices of size at most $k$ such that their deletion results in a graph of treewidth at most 1, i.e., a forest. Currently fastest known deterministic algorithm for the FEEDBACK VERTEX SET problem runs in $3.83^{k} n^{O(1)}$ time $^{1}$ [4]; however, having allowed randomization a $3^{k} n^{O(1)}$ running time can be achieved [5]. A kernel of size $O\left(k^{2}\right)$ is due to Thomassé [22]. Very recently, Philip et al. [18] initiated a study of the parameterized complexity of a closely related problem-PATHWIDTH ONE VERTEX DELETION.

PATHWIDTH ONE VERTEX DELETION (POVD)

Parameter: $k$

Input: An undirected graph $G=(V, E)$ and a positive integer $k$

Question: Does there exist a set $T \subseteq V$ such that $|T| \leq k$ and $G[V \backslash T]$ is a graph of pathwidth at most one?

We omit the formal definitions of pathwidth and treewidth (an interested reader is invited to read, e.g., Diestel's book [7] on graph theory), as the following simple characterisation will be sufficient for our purposes:

Definition 1 A graph is a caterpillar iff it is a tree and after removing all vertices of degree one it becomes a path (possibly empty or consisting of a single vertex). A caterpillar forest is a graph whose every connected component is a caterpillar.

Lemma 1 [2] A graph has pathwidth at most one if and only if it is a caterpillar forest.

\footnotetext{
${ }^{1}$ In this article by $n$ we denote the size of the input graph, i.e., $n=|G|=|V(G)|+|E(G)|$.
} 
The class of caterpillars and caterpillar forests has been studied as further simplifications of trees. A number of problems which have been proved to be difficult even in the class of trees have efficient solutions for caterpillars. Examples include BANDWidth $[3,12,17]$, Proper Interval Colored Graph and Proper Colored LAYOUT [1].

Philip et al. [18] have shown a simple branching algorithm that solves POVD in $7^{k} n^{O(1)}$ time, and a kernel consisting of $O\left(k^{4}\right)$ vertices. In this paper we improve these bounds: we show a $4.65^{k} n^{O(1)}$ FPT algorithm and a kernel consisting of $O\left(k^{2}\right)$ vertices and edges. Let us note that our kernel is not a proper kernel in the following sense: the kernelization algorithm takes a POVD instance $(G, k)$ and outputs an equivalent instance $\left(G^{\prime}, k^{\prime}\right)$ such that $\left|G^{\prime}\right|, k^{\prime} \leq O\left(k^{2}\right)$. However, it may happen that the new parameter $k^{\prime}$ is indeed of size $\Omega\left(k^{2}\right)$. As it may happen that the running time of an algorithm solving the kernelized instance is strongly dependent on $k$ (for instance if one wants to run an FPT algorithm, or mix it with another algorithm in some win-win approach) this may be very undesirable. This motivates a search for proper kernels, ie. kernels in which $\left|G^{\prime}\right| \leq g(k)$ and $k^{\prime} \leq k$. As far as proper kernels are considered, we obtain a cubic kernel $\left(\left|G^{\prime}\right| \leq O\left(k^{3}\right)\right)$ for POVD. We also obtain a smaller, proper quadratic kernel (that is, with $\left|G^{\prime}\right| \leq O\left(k^{2}\right) k^{\prime} \leq k$ ) for a version of the problem which allows multiple edges:

Multigraph Pathwidth One Vertex Deletion (MPOVD) Parameter: $k$ Input: An undirected multigraph $G=(V, E)$ and a positive integer $k$

Question: Does there exist a set $T \subseteq V$ such that $|T| \leq k$ and $G[V \backslash T]$ is a simple graph of pathwidth at most one?

Note that in MPOVD we require that the graph $G[V \backslash T]$ is simple, i.e., the solution is required to hit all multiple edges.

It may appear surprising that we are able to push the proper kernelization upper bounds lower in a seemingly more difficult problem. The reason for this is that in the kernelized MPOVD instance we allow multiple edges, while when performing reductions for POVD they need to be avoided, which turns out to be problematic.

Observe that the first of our kernelization results almost matches known kernel lower bounds for POVD. It follows from a general result of Dell and van Melkebeek [6] that there cannot exist a kernel for POVD that can be encoded in $O\left(k^{\gamma}\right)$ bits for any $\gamma<2$ unless coNP $\subseteq \mathrm{NP} /$ poly, as it may be easily seen that the class of graphs of pathwidth at most one is nontrivial and hereditary (the appropriate definitions are given in the paper [6] stating this result).

Let us also mention that PATHWIDTh ONE VERTEX Deletion problem is closely related to another problem, namely TWO LAYER PLANARIZATION:

TWO LAYER PLANARIZATION (TLP)

Parameter: $k$

Input: An undirected graph $G=(V, E)$ and a positive integer $k$

Question: Does there exist a set $S \subseteq E$ such that $|S| \leq k$ and $(V, E \backslash S)$ is a graph of pathwidth at most one?

In other words, in TLP one can delete at most $k$ edges instead of vertices. The parameterized complexity of TWO LAYER PLANARIZATION has been widely studied. 
Dujmović et al. [9] showed a $6^{k} n^{O(1)}$ algorithm and a kernel with $O(k)$ vertices and edges. The complexity of the algorithm has been further improved to $5.19276^{k} n^{O(1)}$ by Fernau [10], and finally to $3.562^{k} n^{O(1)}$ by Suderman [21]. Recently, Uhlmann and Weller studied TLP parameterized by the size of a minimum feedback edge set [23], i.e., the minimum number of edges that need to be deleted from the graph to obtain a forest. Observe that this parameter is a lower bound on the solution size of TLP, so this is a stronger parameterization. Their results include a kernel with $O(f)$ vertices and edges, as well as an algorithm with running time $6^{f} n^{O(1)}$, where $f$ is the size of a minimum feedback edge set.

Organization In Sect. 2 we introduce some notation and give several introductory observations and results. In Sect. 3 we give a simple $4.65^{k} n^{O(1)}$ branching algorithm that solves POVD. In Sect. 4 we describe the kernelization algorithms. Finally, Sect. 5 contains some concluding remarks and open problems.

\section{Preliminaries}

Notation For a graph or multigraph $G$ we denote by $V(G)$ the set of vertices of the graph. The degree of a vertex $v \in V(G)$ is the number of edge ends incident to it (so a loop contributes 2 to the degree). $K_{3}$ is used to denote the 3 -element cycle, while $C_{4}$ denotes the four-element cycle. A graph $T$ is a tree iff it is connected and contains no cycle. By rooting a tree we mean distinguishing one of its vertex $r$, further called a root. The depth of a rooted tree is the maximum number of vertices on a path connecting the root with a leaf. By $N(v)$ we denote the set of neighbours of the vertex $v$. For $S \subseteq V$, by $N(S)$ we denote $\left(\bigcup_{s \in S} N(s)\right) \backslash S$.

By contracting an edge $u v$ in a multigraph $G$ we mean the following operation. We introduce a new vertex $w$ and remove the edge $u v$ we are contracting. Next, for every edge having $u$ as an endpoint, we substitute the endpoint $u$ with $w$. Similarly we do for $v$. Finally, we remove vertices $u, v$ from the graph. Note that all the loops situated in $u$ or $v$ after the contraction are situated in $w$. Moreover, if there were multiple edges $u v$, after the contraction all but the contracted one become loops situated in $w$. Note also that contraction may introduce new multiple edges in case when $u, v$ had a common neighbour.

The example shown on Fig. 1 presents all the technical details of the used notion of contraction.

Let us recall the characterization of graphs of pathwidth one, proved by Philip et al. [18].

Lemma 2 [18] A simple graph $G$ has pathwidth at most one iff it does not contain cycles and subgraphs isomorphic to the graph $T_{2}$, depicted in Fig. 2.

Fig. 1 Example of a contraction of one of the edges $u v$

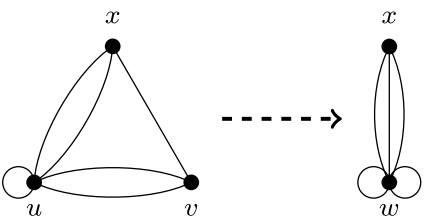


Fig. 2 A forbidden subgraph $T_{2}$ in graphs of pathwidth at most one. The notation for the vertices is used in Lemma 6 and in the proof of Theorem 3

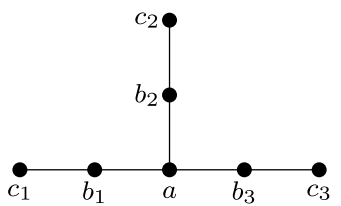

The following corollary of Lemma 2 has been introduced [18] in a slightly different formulation.

Lemma 3 [18] If a simple graph $G$ does not contain $T_{2}, K_{3}$ or $C_{4}$ as subgraphs, then the PATHWIDTH ONE VERTEX DELETION problem on $G$ can be solved in polynomial time.

Proof We begin by introducing the following simple definition:

Definition 2 If $w$ is a vertex of degree 1, and $v$ is its sole neighbour, we call $w$ a pendant of $v$.

Let $G$ be a graph that does not contain $T_{2}, K_{3}$ and $C_{4}$ as subgraphs. Let $H$ be any connected component of $G$. If $H$ is a tree, it does not contain any cycles as subgraphs, and due to Lemma 2 it already has pathwidth at most one. Otherwise, let $C$ be a shortest cycle in $H$. As we excluded $K_{3}$ and $C_{4}$ as subgraphs, $C$ has length at least 5. We aim to prove that all vertices of $H$ either lie on $C$ or are pendants of vertices lying on $C$.

Consider any vertex $v$ on $C$, and any $w$ adjacent to $v$, but not lying on $C$. If $w$ has another neighbour $u \in V(C)$, then the cycle formed by $w$ and the shorter of the arcs connecting $u$ and $v$ on $C$ would be shorter than $C$, a contradiction (as $|V(C)| \geq 5$, the length of the longer arc is at least 3). On the other hand, if $w$ has a neighbour $u \notin V(C)$, then $v, w, u$ and the arcs of length two in both directions from $v$ on $C$ form a $T_{2}$ subgraph of $H$ (the two arcs are disjoint as $|V(C)| \geq 5$ ), again a contradiction. Thus any vertex adjacent to $C$ is a pendant of some vertex of $C$, and as $H$ is connected, there are no other vertices in $H$.

Additionally we know that there are no internal edges (chords) in $C$, since we could get a shorter cycle using such a chord. Now note that any solution of POVD needs to delete at least one vertex of $C$, while the deletion of any vertex of $C$ makes $H$ a caterpillar. Thus, the minimum number of deleted vertices in the graph $G$ is exactly the number of connected components of $G$ that are not trees.

Now we state a simple observation which enables us to add pendant vertices to the input graph.

Lemma 4 If $G$ is a caterpillar forest where a vertex $v \in V(G)$ is of degree at least two, then after adding a pendant vertex $w$ adjacent to $v$ the graph remains a caterpillar forest. 
Proof After adding $w$ the set of non-pendant vertices does not change. Hence the set of non-pendant vertices induces a set of paths. This implies that the graph remains a caterpillar forest.

Lemma 5 If $(G, k)$ is an instance of MPOVD and $u$ is a vertex with at least $k+2$ neighbours in $G$ then $(G, k)$ is equivalent to $\left(G^{\prime}, k\right)$, where $G^{\prime}$ is constructed from $G$ by adding $w$-a pendant neighbour of $u$.

Proof Obviously, the intersection of every solution to MPOVD in $\left(G^{\prime}, k\right)$ with $V(G)$ is a solution to MPOVD in $(G, k)$.

Now take a solution $T \subset V(G)$ to MPOVD in $(G, k)$. If $u \in T$, then $T$ is also a solution to MPOVD in $\left(G^{\prime}, k\right)$. Otherwise, there are at least two neighbours of $u$ not in $T$, thus $u$ is of degree at least 2 in the caterpillar forest $G[V(G) \backslash T]$. But then $G\left[V\left(G^{\prime}\right) \backslash T\right]$ is also a caterpillar forest by Lemma 4, and so $T$ is a solution to MPOVD in $\left(G^{\prime}, k\right)$ also in this case.

Let us now recall two results from matching theory, which will be extensively used in the construction of the kernel:

Theorem 1 (Ore's formula [16], Theorem 1.3 .1 of [13]) Let $H$ be a nonempty bipartite graph with a bipartition $(X, Y)$. Then the maximum cardinality of a matching in $H$ is equal to $|X|-\max _{A \subseteq X} \operatorname{def}(A)$, where $\operatorname{def}(A)=|A|-|N(A)|$. If $A \subseteq X$ is a set which maximizes the value of $\operatorname{def}(A)$, then every maximal matching in $H$ matches $|A|-\operatorname{def}(A)$ vertices from $A$ and all vertices from $X \backslash A$.

Moreover, a set A maximizing the value of $\operatorname{def}(A)$ can be computed in time polynomial in the size of $H$.

Theorem 2 ( $\alpha$-Expansion Lemma, Theorem 2.3 of [22]) Let $H$ be a nonempty bipartite graph with a bipartition $(X, Y)$ with $|Y| \geq \alpha|X|$, where $\alpha$ is a positive integer, and such that every vertex of $Y$ has at least one neighbour in $X$. Then there exist nonempty subsets $X^{\prime} \subseteq X, Y^{\prime} \subseteq Y$ and a function $\phi: X^{\prime} \rightarrow\left(\begin{array}{c}Y^{\prime} \\ \alpha\end{array}\right)$ (the set of subsets of $Y^{\prime}$ of cardinality $\alpha$ ) such that

$-N\left(Y^{\prime}\right)=X^{\prime}$

- $\phi(x) \subseteq N(x)$ for every $x \in X^{\prime}$;

- the sets $\phi(x)$ are disjoint for $x \in X^{\prime}$.

The elements of $\phi(x)$ will be denoted by $y_{1}^{x}, y_{2}^{x}, \ldots, y_{\alpha}^{x}$, and will be jointly referred to as the private neighbours of $x$.

Moreover, such $X^{\prime}, Y^{\prime}$ and $\phi$ can be computed in time polynomial in the size of $H$.

Note that the private neighbours of $x$, the set $\phi(x)$, can be neighbours of any vertex of $X^{\prime}$, but cannot be adjacent to any vertex from $X \backslash X^{\prime}$.

\section{The $4^{k}{ }^{k}{ }^{O(1)}$ Algorithm for POVD}

In this section we develop a $4.65^{k} n^{O(1)}$ branching FPT algorithm for POVD. Firstly, let us recall the approach used by Philip et al. [18]. By Lemma 3 we know that if we 
hit all subgraphs that are isomorphic to $K_{3}, C_{4}$ or $T_{2}$, the problem becomes solvable in polynomial time. Thus, if a graph contains one of the forbidden subgraphs, we branch and guess which of its vertices should be deleted in the solution. There are at most 7 choices (for $T_{2}$ ), thus we obtain a $7^{k} n^{O(1)}$ algorithm described by Philip et al. [18].

Our algorithm uses the same approach, but we use the following combinatorial observation to enhance branching rules for $T_{2}$.

Lemma 6 Let $G$ be a graph and let $H$ be its subgraph isomorphic to $T_{2}$. Denote the vertices of $H$ as in Fig. 2. Assume that the degree of vertex $b_{1}$ in the whole graph $G$ is equal to two. If $(G, k)$ is a YES instance of POVD and there exists a solution A that satisfies $A \cap\left\{a, b_{1}, b_{2}, b_{3}\right\}=\left\{b_{1}\right\}$ then there exists a solution $A^{\prime}$, such that $\left|A^{\prime}\right| \leq|A|$ and $A^{\prime} \cap\left\{a, b_{1}, b_{2}, b_{3}\right\}=\emptyset$.

Proof Assume that we have a solution $A$ to POVD that satisfies $b_{1} \in A, b_{2}, b_{3}$, $a \notin A$. We argue that $A^{\prime}:=A \cup\left\{c_{1}\right\} \backslash\left\{b_{1}\right\}$ is also a valid solution to POVD. As $\left|A^{\prime}\right| \leq|A|$ and $A^{\prime} \cap\left\{a, b_{1}, b_{2}, b_{3}\right\}=\emptyset$, this proves the lemma.

If $G[V \backslash A]$ is a caterpillar forest then obviously $G\left[V \backslash\left(A \cup\left\{c_{1}\right\}\right)\right]$ also is. However, since $\left\{a, b_{2}, b_{3}\right\} \cap A=\emptyset$, we know that the vertex $a$ is of degree at least two in $G[V \backslash A]$. Hence by Lemma 4 we can add a pendant vertex adjacent to $a$ and the graph remains a caterpillar forest. In this way we obtain a graph which is isomorphic to $G\left[V \backslash A^{\prime}\right]$, so $A^{\prime}$ is a valid solution to POVD instance $(G, k)$.

We are now ready to present branching rules that prove the main theorem of this section.

Theorem 3 There exists a $4.65^{k} n^{O(1)}$ FPT algorithm for PATHWIDTH ONE VERTEX DELETION.

Proof As in the algorithm of Philip et al. [18], the algorithm hits all subgraphs isomorphic to $K_{3}, C_{4}$ or $T_{2}$ and then solves the remaining instance in polynomial time using Lemma 3. At each step, the algorithm first looks for subgraphs isomorphic to $K_{3}$ or $C_{4}$ and if it finds one, it guesses which vertex of the forbidden subgraph should be included in the solution. We have at most 4 branches and each branch decreases $k$ by one, thus this branching rule fits into the claimed time bound.

In the rest of the analysis we assume that the girth of the graph $G$ is at least 5 and there exists a subgraph $H$ isomorphic to $T_{2}$. Denote vertices of $H$ as in Fig. 2 . We are going to guess which vertices of $H$ are included in the solution, however we use Lemma 6 to limit the number of choices or to delete more than one vertex in some branches. In order to obtain the claimed time bound in each branching point the recurrence should not be worse than $T(k) \leq 4 T(k-1)+3 T(k-2)$, which leads to a $4.65^{k} n^{O(1)}$ running time since $4 \cdot 4.65^{-1}+3 \cdot 4.65^{-2} \leq 1$. Let us distinguish four cases, depending on how many vertices $b_{i}$ are of degree 2 in $G$.

Case 0 . All vertices $b_{i}$ have degree at least 3 . For $i=1,2,3$ denote by $c_{i}^{\prime}$ any neighbour of $b_{i}$ different than $c_{i}$ and $a$. Since $G$ has girth at least 5, vertices $c_{i}^{\prime}$ are pairwise different and different from vertices in $V(H)$. Let us branch on the following seven 
options. In the first four branches, either $a, b_{1}, b_{2}$ or $b_{3}$ is included in the solution. We have 4 branches, each decreases $k$ by one. In the last three branches both $c_{i}$ and $c_{i}^{\prime}$ are included in the solution for some $i=1,2,3$, hence in each branch $k$ is decreased by two. To prove the correctness of this branching rule note that if we have a solution to POVD that is disjoint with $\left\{a, b_{1}, b_{2}, b_{3}\right\}$ and does not delete both $c_{1}$ and $c_{1}^{\prime}$ nor both $c_{2}$ and $c_{2}^{\prime}$, there are six remaining vertices forming a subgraph isomorphic to $T_{2}$ with $c_{3}$ as well as with $c_{3}^{\prime}$. Thus both $c_{3}$ and $c_{3}^{\prime}$ need to be included in the solution.

Case 1. Exactly one vertex $b_{i}$ (say $b_{1}$ ) has degree 2 . For $i=2,3$ denote by $c_{i}^{\prime}$ any neighbour of $b_{i}$ different than $c_{i}$ and $a$. As before, $c_{2}^{\prime} \neq c_{3}^{\prime}$ and $c_{2}^{\prime}, c_{3}^{\prime} \notin V(H)$. Let us branch on the following six options. In the first four branches, either $a, b_{2}, b_{3}$ or $c_{1}$ is included in the solution. In the fifth branch we include $c_{2}$ and $c_{2}^{\prime}$ in the solution and in the sixth branch we include $c_{3}$ and $c_{3}^{\prime}$. We have four branches that decrease $k$ by one and two that decrease $k$ by two. Let us now check correctness. If $a, b_{2}$ and $b_{3}$ are not included in an optimal solution, we may also assume that $b_{1}$ is not included either, due to Lemma 6. If an optimal solution is disjoint with $\left\{a, b_{1}, b_{2}, b_{3}, c_{1}\right\}$ and does not contain both $c_{2}$ and $c_{2}^{\prime}$, it needs to contain both $c_{3}$ and $c_{3}^{\prime}$, as otherwise it misses a subgraph isomorphic to $T_{2}$.

Case 2. Exactly two vertices $b_{i}$ (say $b_{2}$ and $b_{3}$ ) have degree 2 . Let $c_{1}^{\prime}$ be any neighbour of $b_{1}$ different than $c_{1}$ and $a$; as before, $c_{1}^{\prime} \notin V(H)$. Let us branch on the following six options. In the first four branches, one of $a, b_{1}, c_{2}$ and $c_{3}$ is included in the solution. In the fifth branch, $b_{2}$ and $b_{3}$ are included in the solution. In the sixth branch, $c_{1}$ and $c_{1}^{\prime}$ are included in the solution. We have four branches that decrease $k$ by one and two that decrease $k$ by two. Let us now check correctness. If $a$ and $b_{1}$ are not included in an optimal solution $A$, we may assume that either $b_{2}, b_{3} \in A$ or $b_{2}, b_{3} \notin A$ by Lemma 6. In the first case, we fit into the fifth branch. In the second case, if $c_{2}$ and $c_{3}$ are not included in the solution, both $c_{1}$ and $c_{1}^{\prime}$ are-otherwise a subgraph isomorphic to $T_{2}$ is left.

Case 3. All vertices $b_{i}$ are of degree 2 in $G$. We branch into seven options. In the first four cases, one of $a, c_{1}, c_{2}$ and $c_{3}$ is included in the solution. In the last three cases, one of the subsets of $\left\{b_{1}, b_{2}, b_{3}\right\}$ of size two is included in the solution. We have four branches that decrease $k$ by one and three that decrease $k$ by two, thus we fit into the time bound. To check correctness note that if neither $a$ nor any of the vertices $c_{i}$ is included in the solution, we need to include at least one vertex $b_{i}$. But, due to Lemma 6, any solution has to contain at least two of them.

\section{An $O\left(k^{2}\right)$ Kernel for POVD}

\subsection{A Quadratic Kernel for MPOVD}

Firstly, we focus on a kernelization algorithm for MUlTigRAPH PATHWIDTH ONE VERTEX DELETION that transforms an instance $(G, k)$ of MPOVD into an equivalent instance $\left(G^{\prime}, k^{\prime}\right)$, where $k^{\prime} \leq k$ and $\left|G^{\prime}\right|=O\left(k^{2}\right)$. We provide a set of reduction rules, each of which transforms a MPOVD instance $(G, k)$ into another instance $\left(G^{\prime}, k^{\prime}\right)$ in time polynomial in $|G|+k$, where $\left|G^{\prime}\right|<|G|$ and $k^{\prime} \leq k$. For each of the reduction rules we will check correctness, i.e. that the output instance $\left(G^{\prime}, k^{\prime}\right)$ is a 
MPOVD instance equivalent to $(G, k)$. The kernelization algorithm tries to apply the lowest numbered applicable rule. If none of the rules is applicable, we claim that the size of the multigraph is already bounded by $O\left(k^{2}\right)$. Let $(G, k)$ denote the MPOVD instance we are dealing with.

Before we proceed to the formal description of the rules, let us have a quick overview on the whole procedure. Firstly, we apply all the simple rules already developed by Philip et al. [18]. We use one of them, namely Reduction Rule 4, in order to be able to reduce the high-degree vertices (Reduction Rule 6). Thus, the maximum degree of the graph is bounded linearly in the number of vertices that can be deleted. In this situation every deletion of a vertex can reduce the number of edges by $O(k)$ at most, so at most $O\left(k^{2}\right)$ edges can disappear after deletion of the solution. A caterpillar forest has less edges than vertices, so in the reduced instance $|E| \leq|V|+O\left(k^{2}\right)$, or otherwise the answer is clearly NO. If $|V|,|E|$ are large in comparison with $O\left(k^{2}\right)$, we can closely examine the structure of the instance and find out that in fact one of the reduction rules of Philip et al. [18] is applicable, as the graph has to contain long paths with possible tree-like protrusions attached.

Definition 3 We say that vertices $u, v$ are connected by a multiedge if the number of edges between $u, v$ is at least two. In such a case we say that vertices $u, v$ are incident to a multiedge.

The number of multiedges can be reduced quite easily.

Reduction Rule 1 If there is a loop at a vertex $v$, delete $v$ and decrease $k$ by one. If vertices $u$ and $v$ are connected by $\gamma \geq 3$ edges, delete $\gamma-2$ of them, leaving only two.

Proof (of correctness) Observe that by removing the solution we have to obtain a simple graph. Therefore, each vertex with a loop has to be contained in the solution and every solution has to contain at least one endpoint of a multiedge. Choosing any endpoint to the solution removes the multiedge, so the instance with the multiplicity of edges reduced down to two is equivalent.

Reduction Rule 2 For a vertex $u$ in $G$, if it is connected by multiedges to at least $k+1$ other vertices, then delete $u$ and decrement $k$ by one. The resulting instance is $(G[V(G) \backslash\{u\}], k-1)$.

Proof (of correctness) Observe that if $u$ is connected by multiedges to at least $k+1$ other vertices, then each solution not containing $u$ would need to contain all the (at least $k+1$ ) other endpoints of these edges, a contradiction. Therefore, any solution has to contain $u$.

We follow up with a group of reduction rules already provided by Philip et al. [18].

Reduction Rule 3 [18] If a vertex $u$ in $G$ has two or more pendant neighbours, then delete all but one of these pendant neighbours to obtain $G^{\prime}$. The resulting instance is $\left(G^{\prime}, k\right)$. 
Reduction Rule 4 [18] For a vertex $u$ in $G$, if there is a matching $M$ of size $k+3$ in $G[V(G) \backslash\{u\}]$, where each edge in $M$ has at least one end in $N(u)$, then delete $u$ and decrement $k$ by one. The resulting instance is $(G[V(G) \backslash\{u\}], k-1)$.

Reduction Rule 5 (Rule 5 of Philip et al. [18], rephrased) Let $v_{0}-v_{1}-v_{2}-\cdots-$ $v_{p}-v_{p+1}$ be a path in $G$, such that for each vertex $v_{i}(1 \leq i \leq p)$ its neighbours other than $v_{i-1}, v_{i+1}$ are pendant, and $v_{i}$ is not incident to any multiedges. If $p \geq 5$, then contract the edge $\left(v_{2}, v_{3}\right)$ in $G$ to obtain the graph $G^{\prime}$. The resulting instance is $\left(G^{\prime}, k\right)$.

The correctness of the above rules has already been proved by Philip et al. [18]. It is easy to observe that the rules are also correct in the multigraph model we are working with.

Let us now take some integer $\alpha \geq 5$. In this section we use $\alpha=5$, while in the next section $\alpha=\max \{k+2,5\}$ is going to be considered. Our next goal is to bound the degrees of vertices in $G$. The following lemma is crucial for the degree reduction rule.

Lemma 7 Let $\alpha \geq 5$. Consider a MPOVD instance $(G, k)$. Assume that there is a vertex $u \in V(G)$ of degree at least $(5 k+7)+\alpha(k+2)$ and Reduction Rules $1-5$ cannot be applied. Then one can in polynomial time find disjoint sets of vertices $X^{\prime}, Y^{\prime} \subseteq V(G) \backslash\{u\}$ and a function $\phi: X^{\prime} \rightarrow\left(\begin{array}{c}Y^{\prime} \\ \alpha\end{array}\right)$ satisfying the following properties:

1. every vertex in $Y^{\prime}$ is connected by a single edge to $u$,

2. $Y^{\prime}$ is an independent set in $G$,

3. $N\left(Y^{\prime}\right)=X^{\prime} \cup\{u\}$, and each vertex from $Y^{\prime}$ has a neighbour in $X^{\prime}$,

4. the sets $\phi(x) \subseteq N(x)$ are disjoint for any two different $x \in X^{\prime}$ (as before we denote the elements of $\phi(x)$ by $y_{1}^{x}, y_{2}^{x}, \ldots, y_{\alpha}^{x}$ in $Y^{\prime}$ and call them the private neighbours of $x)$.

Proof Before we proceed to the technical details, let us have an informal glimpse on the outline of the proof. Assume that $u$ has a $\operatorname{large}$ degree. If $\operatorname{deg}(u)$ is sufficiently large, then by firstly forgetting all its neighbours adjacent via multiple edges (there are not so many of them, as Reduction Rule 2 is not applicable) and secondly forgetting endpoints of maximum matching of the graph induced by the neighbours (it has to be relatively small, as Reduction Rule 4 was not applicable), we obtain a large independent set of neighbours of $u$, connected via single edges. Now we consider the second neighbourhood, of $u$, namely the neighbours of this independent set, and the bipartite graph between the first end the second neighbourhood induced by edges of $G$. As Reduction Rule 4 was not applicable, the matching in this graph is relatively small; therefore, by Theorem 1 we can find a set with a small deficit. Thus we constructed a large independent set of neighbours of $u$, which know only a small number of vertices in the second neighbourhood. Now we need only to apply Theorem 2 in order to finish the proof.

Let us now transform this intuition into a formal argument.

We invite the reader to read the presented proof while carefully studying Fig. 3, which pictures the details. 


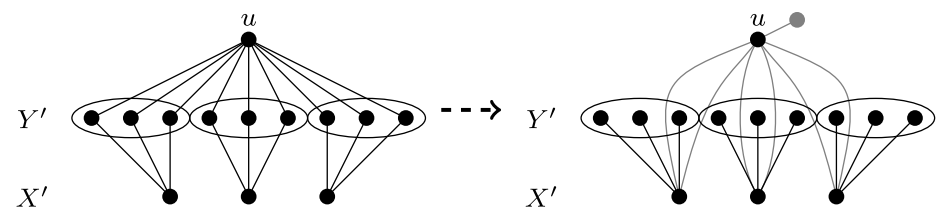

Fig. 3 The situation from Lemma 7 before and after application of the Reduction Rule 6

Let $Y_{0}$ be the set of nonpendant neighbours of $u$, connected to $u$ by single edges. As Reduction Rules 1, 2 and 3 could not be applied, there are at most $2 k+1$ edges incident to $u$ and not incident to $Y_{0}$ : at most $2 k$ which are multiple edges and at most one for a pendant, thus $\left|Y_{0}\right| \geq(\alpha+3)(k+2)$.

Now, let $M_{0}$ be a maximum matching in $G\left[Y_{0}\right]$, constructed in polynomial time [14]. Note that as Reduction Rule 4 could not be applied, $\left|M_{0}\right| \leq k+2$. Let us denote by $Y_{1}$ the set $Y_{0}$ excluding the endpoints of $M_{0}$. As $M_{0}$ was a maximum matching in $G\left[Y_{0}\right], Y_{1}$ is an independent set in $G$ of cardinality at least $(\alpha+1)(k+2)$.

Let us construct a bipartite graph $H_{1}$ on the bipartition $\left(Y_{1}, X_{1}\right)$, where $X_{1}=$ $N\left(Y_{1}\right) \backslash\{u\}$. Vertices $x \in X_{1}$ and $y \in Y_{1}$ are connected by an edge in $H_{1}$ iff they were adjacent in $G$. Let $Y \subseteq Y_{1}$ be a set with the maximum $\operatorname{def}(Y)$ in $H_{1}$ among all subsets of $Y_{1}$, constructed in polynomial time using Theorem 1. Denote $X=N_{H_{1}}(Y)$. Observe that a maximum matching in $H_{1}$ has cardinality $\left|Y_{1}\right|-|Y|+|X|$. As Reduction Rule 4 could not be applied, $k+2 \geq\left|Y_{1}\right|-|Y|+|X|$. As $Y \subseteq Y_{1}$, we conclude that $\left|Y_{1} \backslash Y\right| \leq k+2$ and $|X| \leq k+2$. Thus $|Y| \geq \alpha(k+2)$.

Denote by $H$ the subgraph of $H_{1}$ induced by $X \cup Y$. As $Y_{1}$ was an independent set and all the vertices from $Y_{1}$ were nonpendant neighbours of $u$, by the definition of $X$ every $y \in Y$ has at least one neighbour in $X$. Moreover, $|Y| \geq \alpha(k+2) \geq \alpha|X|$. Using Theorem 2 in polynomial time we construct nonempty sets $Y^{\prime} \subseteq Y, X^{\prime} \subseteq X$ and a function $\phi: X^{\prime} \rightarrow\left(\begin{array}{c}Y^{\prime} \\ \alpha\end{array}\right)$ such that $N\left(Y^{\prime}\right) \cap X=X^{\prime}$ and each $x \in X^{\prime}$ has a set $\phi(x)=\left\{y_{1}^{x}, y_{2}^{x}, \ldots, y_{\alpha}^{x}\right\}$ of $\alpha$ private neighbours in $Y^{\prime}$.

Reduction Rule 6 Let $u$ be a vertex of degree at least $(5 k+7)+\alpha(k+2)$. Let $X^{\prime}$ and $Y^{\prime}$ be the nonempty sets found using Lemma 7. Obtain $G^{\prime}$ by deleting all the edges between $u$ and $Y^{\prime}$ and adding double edges between $u$ and every vertex from $X^{\prime}$. Also, if $u$ had no pendant neighbour, add one. The resulting instance is $\left(G^{\prime}, k\right)$.

Proof (of correctness) Firstly, note that during application of the reduction rule we delete at least $\alpha\left|X^{\prime}\right|$ edges and add at most $2\left|X^{\prime}\right|+1$ edges and one vertex, thus (as $\alpha \geq 5$ and $\left|X^{\prime}\right| \geq 1$ ) strictly reducing the graph size. Now let us prove the correctness of this reduction rule.

We have to show that the resulting instance $\left(G^{\prime}, k\right)$ is equivalent to the input instance $(G, k)$. Observe that the degree of $u$ in $G$ is at least $(5 k+7)+\alpha(k+2)$, so by Lemma 5 one can add a pendant vertex to $u$ without changing the answer to the POVD problem. Thus, without loss of generality we may assume that the pendant vertex has already been attached to $u$ and therefore there are no additional vertices in $G^{\prime}$. Denote this additional pendant vertex by $w$. Observe that one may assume that $w$ is not contained in the solution to POVD in $(G, k)$ and in $\left(G^{\prime}, k\right)$, as choosing 
$w$ to the solution can always be substituted with choosing $u$ without increasing the solution size.

Denote $V=V(G)=V\left(G^{\prime}\right)$ and $l=\left|X^{\prime}\right|$. Suppose that there is a set $A \subseteq V$ such that $|A| \leq k$ and $G[V \backslash A]$ has pathwidth at most one. As $\alpha \geq 2$ then one can find $l$ cycles of length 4 that meet only at $u$ : for each $x \in X^{\prime}$ we form a cycle consisting of $x, y_{1}^{x}, u$ and $y_{2}^{x}$. Observe that if $u \in A$ then $G[V \backslash A]=G^{\prime}[V \backslash A]$ and $A$ is also a solution to MPOVD in $\left(G^{\prime}, k\right)$. Now suppose that $u \notin A$. In this situation, the set $A$ has to contain at least $l$ vertices from $X^{\prime} \cup Y^{\prime}$ in order to have at least one vertex on each of the indicated cycles. Obtain $A^{\prime}=\left(A \backslash Y^{\prime}\right) \cup X^{\prime}$ by replacing these vertices with simply the set $X^{\prime}$ (of cardinality $l$ ). Note that thus $\left|A^{\prime}\right| \leq k$. We claim that $A^{\prime}$ is also a solution to $(G, k)$ instance of MPOVD. Indeed, as $G[V \backslash A]$ has pathwidth at most one, then $G\left[V \backslash\left(A \cup X^{\prime}\right)\right]$ has pathwidth at most one as well. Observe that $G\left[V \backslash A^{\prime}\right]$ can obtained from $G\left[V \backslash\left(A \cup X^{\prime}\right)\right]$ by introducing vertices from $Y^{\prime} \cap A$ as pendant neighbours of $u$. As we argued, we may assume that $u$ already has a pendant neighbour $w$ in $G\left[V \backslash\left(A \cup X^{\prime}\right)\right]$, hence adding additional pendants to $u$ cannot increase the pathwidth. Thus $G\left[V \backslash A^{\prime}\right]$ has also pathwidth at most 1 . Finally, observe that $A^{\prime}$ is also a solution in $\left(G^{\prime}, k\right)$, as $G^{\prime}\left[V \backslash A^{\prime}\right]$ is a graph obtained from $G\left[V \backslash A^{\prime}\right]$ by removing all the edges from $u$ to $Y^{\prime}$. Therefore $\left(G^{\prime}, k\right)$ is a YES instance of MPOVD as well.

Now suppose that $B$ is a solution to MPOVD in $\left(G^{\prime}, k\right)$. Recall that both in $G^{\prime}$ and $G$ the vertex $u$ has a pendant neighbour $w$ and we may assume that $w \notin B$. As before, if $u \in B$, then $G^{\prime}[V \backslash B]=G[V \backslash B]$ and $B$ is also a solution to MPOVD in $(G, k)$. Now suppose that $u \notin B$. As $u$ is connected to all the vertices from $X^{\prime}$ by double edges, $X^{\prime} \subseteq B$. Therefore, in $G[V \backslash B]$ the set $Y^{\prime} \backslash B$ is a set of pendant neighbours of $u$. Observe that $G[V \backslash B]$ can be obtained from $G^{\prime}[V \backslash B]$ by introducing vertices from $Y^{\prime} \backslash B$ as pendant neighbours of $u$ instead of isolated vertices. As there already exists a pendant neighbour $w$ of $u$ in $G^{\prime}[V \backslash B]$, this operation cannot increase the pathwidth. Hence $B$ is a solution to MPOVD in $(G, k)$ as well.

Let us denote $\beta=(5 k+7)+\alpha(k+2)$. Using Reduction Rule 6 we have already bounded the maximum degree by $\beta$. Let us introduce two more reduction rules in order to make the graph denser and thus bound its size.

Reduction Rule 7 Let $u$ be a vertex which is not contained in any cycle or $T_{2}$. Obtain $G^{\prime}$ by deleting $u$. The resulting instance is $\left(G^{\prime}, k\right)$.

Proof (of correctness) Consider any induced subgraph $H$ of $G$ containing $u$. Then $u$ is not contained in any cycle or $T_{2}$ in $H$. We claim that $G[V(H) \backslash\{u\}]$ is a caterpillar forest if and only if $H$ is a caterpillar forest. To see this, observe that if $G[V(H) \backslash\{u\}]$ is a caterpillar forest, then introduction of $u$ cannot create new cycles nor $T_{2}$-s in $H$. The fact that a subgraph of a caterpillar forest is a caterpillar forest concludes the proof of the equivalence.

This implies that a set $A \subseteq V(G)$ is a solution to MPOVD in $(G, k)$ iff $A \backslash\{u\}$ is a solution. Thus $u$ is not contained in any minimal solution, and a set $A$ is a minimal solution to MPOVD in $(G, k)$ iff it is a minimal solution in $\left(G^{\prime}, k\right)$. 
The next reduction rule can be viewed as greedy deletion of vertices in treelike parts of the graph.

Reduction Rule 8 Let $W \subseteq V(G)$ be a set of vertices such that $G[W]$ is a tree (without loops or multiple edges) containing at least one $T_{2}$ and there are no edges between $W \backslash\{w\}$ and $V(G) \backslash W$ for some $w \in W$. Root $G[W]$ in $w$ and in every $T_{2}$ contained in $G[W]$ mark the vertex nearest to $w$. Let $c$ be the vertex farthest from $w$ among the marked ones. Delete $c$ to obtain $G^{\prime}$ and decrement $k$ by one. The resulting instance is $\left(G^{\prime}, k-1\right)$.

Proof (of correctness) Let $F$ be a $T_{2}$ subgraph of $G[W]$, due to which $c$ has been marked. Observe that at least one of the vertices from $V(F)$ has to be deleted and that the deletion of any such vertex can be substituted by the deletion of $c$-every $T_{2}$ hit by a vertex from $V(F)$ is also hit by $c$, due to extremality of $c$ and $F$. Thus one can safely assume that $c$ is in the solution.

Note that the situation described in Reduction Rule 8 can be recognized in polynomial time-for every vertex $w$ one deletes $w$ and chooses as $W$ the union of $w$ and these connected components derived from the connected component in which $w$ was, which are trees.

Now we claim that if none of the Reduction Rules 1-8 can be applied and there is a solution to MPOVD in $(G, k)$ then the size of $G$ is bounded by $O\left(k^{2}\right)$.

Lemma 8 Let $(G, k)$ be a YES instance of MPOVD. If Reduction Rules 1-8 are not applicable, then $|V(G)| \leq(220.5 \beta+63) k$ and $|E(G)| \leq(252 \beta+72) k$.

Proof Take any vertex $w$. Let $G_{w}$ be the connected component of $w$ in $G$. Let $H_{1}, H_{2}, \ldots, H_{\ell}$ be those connected components of $G_{w}\left[V\left(G_{w}\right) \backslash\{w\}\right]$ that are trees (without any loops or multiple edges) and for each $H_{i}(1 \leq i \leq \ell)$ there is exactly one edge between $H_{i}$ and $w$. We claim that $H_{1}, H_{2}, \ldots, H_{\ell}$ have at most 6 vertices in total. Let $W_{w}$ be $\{w\} \cup \bigcup_{i=1}^{l} V\left(H_{i}\right) . G\left[W_{w}\right]$ is a tree not satisfying the assumptions of Reduction Rule 8, so $G\left[W_{w}\right]$ does not contain any $T_{2}$. On the other hand, as Reduction Rule 7 was not applicable, any vertex $v \in W_{w} \backslash\{w\}$ has to be contained in some $T_{2}$. Thus the depth of $G\left[W_{w}\right]$ after rooting in $w$ is at most 3-otherwise the deepest leaf would not be in any cycle nor $T_{2}$ and Reduction Rule 7 would be applicable.

Assume at first that $w$ is a pendant in $G\left[W_{w}\right]$. Then $w^{\prime}$, the only neighbour of $w$ in $G\left[W_{w}\right]$, has got at most 3 neighbours other than $w$ : at most one other pendant and at most two non-pendant (if there were three of them, there would be a $T_{2}$ in $\left.G\left[W_{w}\right]\right)$. These non-pendant neighbours can have only pendant other neighbours, as otherwise the depth of the tree would exceed 3. Therefore, they have at most one other neighbour that is pendant, resulting in $G\left[W_{w}\right]$ having at most 7 vertices.

Now assume that $w$ has at least two neighbours. Similarly as before, $w$ has at most 3 neighbours in $G\left[W_{w}\right]$ at all: one pendant and two non-pendant. Let $w^{\prime}$ be any non-pendant neighbour of $w$. Observe that apart from $w, w^{\prime}$ can have at most one pendant neighbour and at most one non-pendant neighbour-two non-pendant 
neighbours would result in a $T_{2}$ with the centre in $w^{\prime}$ and one leg passing through $w$. Assume for a moment that such a non-pendant neighbour of $w^{\prime}$ exists and denote it by $w^{\prime \prime}$. Observe that a $T_{2}$ containing a neighbour of $w^{\prime \prime}$ other than $w^{\prime}$ (there is one, as otherwise Reduction Rule 7 would be applicable) has to be entirely contained in $G\left[W_{w}\right]$, as it cannot have center in $w^{\prime}$. We obtained a contradiction with the assumption that $G\left[W_{w}\right]$ contained no $T_{2}$-s entirely. Therefore, every non-pendant neighbour of $w$ has at most one other neighbour that is a pendant. As $w$ has at most three neighbours in $G\left[W_{w}\right]$ : at most one pendant and at most two non-pendant, we infer that in this case $G\left[W_{w}\right]$ has at most 6 vertices.

Now we proceed to the simplification of the graph $G$. Consider the following procedure: while there exists a vertex of degree at most 1 in $G$, remove it. Denote the remaining set of vertices as $V_{1}$. Denote $H_{1}=G\left[V_{1}\right]$. Observe that the construction of $H_{1}$ can only decrease the degrees of vertices in $V_{1}$, so the degrees of vertices in $H_{1}$ are also bounded by $\beta$, as Reduction Rule 6 was not applicable.

As Reduction Rules 7, 8 could not be applied, no connected component of $G$ is a tree. Hence each vertex $v \in V(G) \backslash V_{1}$ belongs to the tree $G\left[W_{w}\right]$ for the vertex $w \in V_{1}$ that is closest to $v$ (there is exactly one such vertex). Thus every vertex $v \in$ $V(G) \backslash V_{1}$ belongs to a tree $G\left[W_{w}\right]$ for some $w \in V_{1}$. The trees $G\left[W_{w}\right]$ for $w \in V_{1}$ are disjoint, so $|V(G)| \leq 7\left|V_{1}\right|$ and $|E(G)|=\left|E\left(H_{1}\right)\right|+|V(G)|-\left|V_{1}\right|$.

Consider any walk $v_{1}, v_{2}, \ldots, v_{9}$ in $H_{1}$ where the inner 7 vertices have degree 2 in $H_{1}$. The vertices $v_{1}$ and $v_{9}$ possibly coincide, but all the inner vertices are distinct from each other and from $v_{1}$ and $v_{9}$. If $v_{3}, v_{4}, v_{5}, v_{6}$ and $v_{7}$ all had only pendant neighbours in $V(G) \backslash V_{1}$, we could apply Reduction Rule 5, a contradiction. Thus one of them (say $v_{i}$ ) has a neighbour $w_{1} \in V(G) \backslash V_{1}$, which has a neighbour $w_{2} \in V(G) \backslash V_{1}$. Therefore, $v_{i}$ is the " $a$ " vertex of a $T_{2}$ (see Fig. 2), as $v_{i-2}, v_{i-1}, v_{i}, v_{i+1}, v_{i+2}, w_{1}, w_{2}$ form a $T_{2}$. Thus we can have at most $k$ such vertexdisjoint 9-vertex walks-for otherwise we would obtain $k+1$ vertex-disjoint $T_{2}$ subgraphs, a contradiction.

Let $H_{2}$ be a multigraph with possible loops, obtained from $H_{1}$ as follows: while there is any vertex of degree exactly 2 , we contract one of the edges incident to this vertex. Observe that when contracting an edge incident to a vertex $v$ of degree 2, all the degrees of vertices not incident to the edge stay the same. Moreover, the degree of the vertex obtained in the contraction is equal to the degree of the second endpoint, different from $v$. In $H_{1}$ there were no vertices of degree 0 nor 1 ; therefore, in $H_{2}$ every vertex either is isolated and has a self-loop or has degree at least 3 . The edges in $H_{2}$ correspond to paths in $H_{1}$, in which every inner vertex has degree 2 (possibly there are no inner vertices on such a path; a loop corresponds to a path in which the first and last vertex coincide). Let $s_{e}$ be the number of edges on the path in $H_{1}$ corresponding to $e \in E\left(H_{2}\right)$. Consider any edge $e$ with $s_{e} \geq 10$. In the corresponding path we can find $\left\lfloor\frac{s_{e}-1}{9}\right\rfloor$ entirely contained vertex-disjoint subpaths with 9 vertices each. As we have argued, we have at most $k$ such subpaths at all, hence

$$
\sum_{e \in E\left(H_{2}\right)} \frac{s_{e}-1}{9} \leq \sum_{e \in E\left(H_{2}\right)}\left(\left\lfloor\frac{s_{e}-1}{9}\right\rfloor+\frac{8}{9}\right) \leq k+8\left|E\left(H_{2}\right)\right| / 9 .
$$


As each edge in $E\left(H_{1}\right)$ is contained in some path corresponding to an edge in $E\left(H_{2}\right)$, we obtain

$$
\left|E\left(H_{1}\right)\right|=\sum_{e \in E\left(H_{2}\right)} s_{e}=\left(9 \sum_{e \in E\left(H_{2}\right)} \frac{s_{e}-1}{9}+\left|E\left(H_{2}\right)\right|\right) \leq 9\left|E\left(H_{2}\right)\right|+9 k .
$$

Let $A$ be a solution to MPOVD in $(G, k)$. We may assume that $A \subseteq V_{1}$, as if $v \in V(G) \backslash V_{1}$ and $v \in W_{w}$, we may take $w$ into $A$ instead of $v$ (as in the proof of correctness of Reduction Rule 8). Construct $A^{\prime} \subseteq V\left(H_{2}\right)$ as the image of $A$ in the contractions creating $\mathrm{H}_{2}$ (i.e., if we contract an edge, where at least one of the endpoints was in $A$, the resulting vertex is in $A^{\prime}$ ). Observe that $A^{\prime}$ is a solution to MPOVD in $\left(H_{2}, k\right)$-we have $\left|A^{\prime}\right| \leq|A|$, and any cycle or $T_{2}$ in $G\left[V\left(H_{2}\right) \backslash A^{\prime}\right]$ would correspond to a cycle or $T_{2}$ in $G[V(G) \backslash A]$. Moreover, the contractions could not increase the maximum degree in $\mathrm{H}_{2}$, so the maximum degree in $\mathrm{H}_{2}$ is also bounded by $\beta$, as Reduction Rule 6 was not applicable. Let us recall that in $H_{2}$ every vertex either is isolated and has a self-loop or has degree at least 3. Let $H_{2}^{1}$ be the subgraph of $H_{2}$ made up of isolated vertices with self-loops and $H_{2}^{2}$ be the subgraph of $H_{2}$ made up of all the connected components containing at least two vertices. By this definition, every vertex in $H_{2}^{2}$ is of degree at least three and $V\left(H_{2}^{1}\right) \subseteq A^{\prime}$ since self-loops need to be hit, hence $\left|V\left(H_{2}^{1}\right)\right| \leq\left|A^{\prime}\right| \leq k$. Furthermore, the degrees of vertices in both the graphs $H_{2}^{1}, H_{2}^{2}$ are bounded by $\beta$, hence $\left|E\left(H_{2}^{1}\right)\right| \leq \beta k / 2$ (note that it is possible for a vertex in $H_{2}^{1}$ to have multiple loops, so we cannot use the trivial bound $\left.\left|E\left(H_{2}^{1}\right)\right| \leq k\right)$. Moreover, after the deletion of vertices from $A^{\prime}$ the multigraph $H_{2}^{2}$ becomes a forest. Consequently, we can partition the edges in $H_{2}^{2}$ into two sets: those deleted by the deletion of $A^{\prime}$ (at most $\beta k$ of them) and those contained in the resulting forest (at most $\left|V\left(H_{2}^{2}\right)\right|$ of them). Vertices in $H_{2}^{2}$ have degree at least 3, so

$$
\frac{3}{2}\left|V\left(H_{2}^{2}\right)\right| \leq\left|E\left(H_{2}^{2}\right)\right| \leq\left|V\left(H_{2}^{2}\right)\right|+\beta k .
$$

Thus $\left|V\left(H_{2}^{2}\right)\right| \leq 2 \beta k$ and $\left|E\left(H_{2}^{2}\right)\right| \leq 3 \beta k$. We can now bound the number of edges of the graph $H_{2}:\left|E\left(H_{2}\right)\right|=\left|E\left(H_{2}^{1}\right)\right|+\left|E\left(H_{2}^{2}\right)\right| \leq 7 \beta k / 2$. As a result, $\left|E\left(H_{1}\right)\right| \leq$ $9\left|E\left(H_{2}\right)\right|+9 k \leq(63 \beta / 2+9) k$. Note that $\left|E\left(H_{2}^{1}\right)\right| \geq\left|V\left(H_{2}^{1}\right)\right|$, as each vertex from $V\left(H_{2}^{1}\right)$ has at least one loop. As vertices in $V\left(H_{2}^{2}\right)$ have degree at least $3,\left|E\left(H_{2}^{2}\right)\right| \geq$ $\frac{3}{2}\left|V\left(H_{2}^{2}\right)\right|$. Hence $\left|E\left(H_{2}\right)\right| \geq\left|V\left(H_{2}\right)\right|$. Now observe that $\left|E\left(H_{1}\right)\right|-\left|V\left(H_{1}\right)\right|=$ $\left|E\left(H_{2}\right)\right|-\left|V\left(H_{2}\right)\right|$, as each contraction removes one edge and one vertex. Therefore,

$$
\left|V\left(H_{1}\right)\right| \leq\left|E\left(H_{1}\right)\right| \leq(63 \beta / 2+9) k .
$$

Now we can bound the number of vertices of $G$ :

$$
|V(G)| \leq 7\left|V\left(H_{1}\right)\right| \leq 7(63 \beta / 2+9) k=(220.5 \beta+63) k,
$$

as well as the number of edges:

$$
|E(G)| \leq\left|E\left(H_{1}\right)\right|+|V(G)| \leq(63 \beta / 2+9) k+(220.5 \beta+63) k=(252 \beta+72) k \text {. }
$$


This lemma justifies the final reduction rule.

Reduction Rule 9 If $|E(G)|>(252 \beta+72) k$ or $|V(G)|>(220.5 \beta+63) k$, the resulting instance is $\left(K_{3}, 0\right)$, which is a trivial NO instance of MPOVD.

Taking $\alpha=5$ (and thus $\beta=10 k+17$ ) we obtain the kernelization algorithm for MPOVD.

Theorem 4 There exists a kernelization algorithm for MULTIGRAPH PATHWIDTH ONE VERTEX DELETION that from an instance $(G, k)$ produces an equivalent instance $\left(G^{\prime}, k^{\prime}\right)$ satisfying $k^{\prime} \leq k,\left|E\left(G^{\prime}\right)\right| \leq 2520 k^{2}+4356 k$ and $\left|V\left(G^{\prime}\right)\right| \leq 2205 k^{2}+$ $3811.5 k$, i.e., $\left|G^{\prime}\right|=O\left(k^{2}\right)$.

\subsection{Kernels for POVD}

Firstly, let us establish an equivalence between POVD and MPOVD instances. Obviously, any instance of POVD can be treated as an instance of MPOVD. In the other direction, the following lemma shows that each multiedge can be replaced by a small gadget at the cost of a small increase of the parameter.

Lemma 9 Let $(G, k)$ be an MPOVD instance. One can compute in $|G|^{O(1)}$ time complexity an equivalent instance $\left(G^{\prime}, k^{\prime}\right)$ of POVD, where $\left|G^{\prime}\right|=O(|G|)$ and $k^{\prime} \leq$ $k+|E(G)|$.

Proof Firstly, if there exists a vertex $v$ with a loop, we remove this vertex and decrease $k$ by one, since this vertex has to be contained in any solution. Next, for each pair of vertices $v_{1}, v_{2} \in V(G)$ connected by a multiedge, we replace all edges $v_{1} v_{2}$ with the gadget shown on Fig. 4 and increase the parameter by one. The new instance $\left(G^{\prime}, k^{\prime}\right)$ clearly satisfies $\left|G^{\prime}\right|=O(|G|)$ and $k^{\prime} \leq k+|E(G)|$. Let us now check the correctness of this construction. Obviously, we need to check only the correctness of a single replacement. Let us assume that $v_{1}$ and $v_{2}$ are connected by a multiedge in the instance $(G, k)$ and the instance $\left(G^{\prime}, k+1\right)$ is created by replacing the multiedge $v_{1} v_{2}$ with the gadget. We now argue that these instances are equivalent.

Assume that $(G, k)$ is a YES instance and let $A \subseteq V(G)$ be a valid solution. If $\left\{v_{1}, v_{2}\right\} \subseteq A$, then $A \cup\{u\}$ is a valid solution to $\left(G^{\prime}, k+1\right)$. Otherwise, as $A \cap\left\{v_{1}, v_{2}\right\} \neq \varnothing$, w.l.o.g. assume that $v_{1} \in A$ and $v_{2} \notin A$. Then $A \cup\left\{w_{2}\right\}$ is a valid solution to $\left(G^{\prime}, k+1\right)$.

Now assume that $\left(G^{\prime}, k+1\right)$ is a YES instance and let $A^{\prime} \subseteq V\left(G^{\prime}\right)$ be a valid solution. Denote $X=\left\{c_{1}, c_{2}, b_{1}, b_{2}, u, w_{1}, w_{2}, v_{1}, v_{2}\right\}$. Note that $X \backslash\left\{w_{1}, v_{1}\right\}$ and

Fig. 4 A gadget that replaces a multiedge $v_{1} v_{2}$
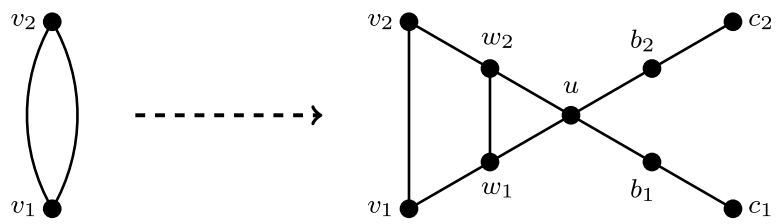
$X \backslash\left\{w_{2}, v_{2}\right\}$ form two subgraphs isomorphic to $T_{2},\left\{v_{1}, v_{2}, w_{1}, w_{2}\right\}$ is a 4-cycle in $G^{\prime}$ and those three forbidden subgraphs have an empty intersection. Thus $\left|A^{\prime} \cap X\right| \geq 2$. If $\left|A^{\prime} \cap X\right| \geq 3$, then $A^{\prime \prime}:=A^{\prime} \backslash X \cup\left\{v_{1}, v_{2}, u\right\}$ is also a valid solution to $\left(G^{\prime}, k+1\right)$ and $A^{\prime \prime} \cap V(G)$ is a valid solution to $(G, k)$. Otherwise, if $\left|A^{\prime} \cap X\right|=2$ note that $A^{\prime} \cap X \neq\left\{v_{1}, v_{2}\right\}$, because otherwise a 3-cycle $u, w_{1}, w_{2}$ is left untouched. W.l.o.g. assume that $v_{1} \notin A^{\prime}$. Then $A^{\prime \prime}:=A^{\prime} \backslash X \cup\left\{v_{2}, w_{1}\right\}$ is a valid solution to $\left(G^{\prime}, k+1\right)$ and $A^{\prime \prime} \cap V(G)$ is a valid solution to $(G, k)$.

We are now ready to conclude with the kernelization results for POVD.

Theorem 5 There exists a kernelization algorithm for PATHWIDTH ONE VERTEX DELETION that from an instance $(G, k)$ produces an equivalent instance $\left(G^{\prime}, k^{\prime}\right)$ satisfying $\left|G^{\prime}\right|+k^{\prime}=O\left(k^{2}\right)$.

Proof At first, treat the instance $(G, k)$ as a MPOVD instance and obtain a MPOVD kernel with $O\left(k^{2}\right)$ vertices and edges. Then use Lemma 9 to obtain a POVD instance $\left(G^{\prime}, k^{\prime}\right)$ with $O\left(k^{2}\right)$ vertices and edges and with $k^{\prime}=O\left(k^{2}\right)$.

Theorem 6 There exists a kernelization algorithm for PATHWIDTH ONE VERTEX DELETION that from an instance $(G, k)$ produces an equivalent instance $\left(G^{\prime}, k^{\prime}\right)$ satisfying $k^{\prime} \leq k$ and $\left|G^{\prime}\right|=O\left(k^{3}\right)$.

Proof We use the same reduction rules as for the MPOVD kernel in Sect. 4.1. Notice that Reduction Rule 6 is the only one which introduces multiedges. Thus, we need to modify it so that it will use only single edges. We proceed as in Reduction Rule 6, however we will use $\alpha=\max \{k+2,5\}$. Let vertex $u$ be of degree at least $(5 k+7)+$ $\alpha(k+3)$, obtain sets $X^{\prime}$ and $Y^{\prime}$ from Lemma 7, remove all edges between $u$ and $Y^{\prime}$ and add a pendant to $u$. However, instead of connecting $u$ to $X^{\prime}$ using double edges, for each $x \in X^{\prime}$ we introduce a new vertex $v_{x}$ and connect vertices $x, v_{x}, u$ into a triangle.

Let us now verify the correctness of the reduction rule. As in the analysis of Reduction Rule 6, due to Lemma 5 we may assume that the pendant vertex has already been attached to $u$ and therefore there are no additional vertices in $G^{\prime}$.

Firstly, let $A$ be a valid solution in the modified $\left(G^{\prime}, k\right)$ instance. We may assume that $A$ does not contain any vertex $v_{x}$. Indeed, as $u$ already has a pendant in $G^{\prime}$, we may delete the vertex $x$ instead of $v_{x}$. Since $u, v_{x}, x$ is a cycle in $G^{\prime}$ for each $x \in X^{\prime}$, either $u \in A$ or $X^{\prime} \subseteq A$. If $u \in A$, then $G^{\prime}\left[V\left(G^{\prime}\right) \backslash A\right]$ is a supergraph of the graph $G[V(G) \backslash A]$ and, thus, $A$ is a solution to the instance $(G, k)$. Otherwise, as in the analysis of Reduction Rule 6, $G[V(G) \backslash A]$ is isomorphic to a subgraph of $G^{\prime}\left[V\left(G^{\prime}\right) \backslash A\right]$ but with $Y^{\prime} \backslash A$ being pendant neigbours of $u$ instead of isolated vertices. However $u$ already has a pendant neighbour in $G^{\prime}$, about which we can assume that it is not contained in $A$, so introduction of these pendant neighbours cannot increase the pathwidth.

Now let $A$ be a valid solution to the instance $(G, k)$. As in the analysis of Reduction Rule 6, either $u \in A$ or $X^{\prime} \subseteq A$. In our case the graph $G^{\prime}\left[V\left(G^{\prime}\right) \backslash A\right]$ differs from the same graph in the MPOVD case by vertices $v_{x}$, which are now pendants of vertex 
$u$ or $x$. Since $\alpha \geq k+2$, vertices $u$ and $x$ have at least $k+2$ neighbours and, due to Lemma 5 , the answer does not change if we add pendant neighbours to them. Thus, the reduction rule is correct.

Clearly, the application of the reduction rule does not increase the parameter. Taking $\alpha=\max \{k+2,5\}$ and, thus, $\beta=O\left(k^{2}\right)$ in Lemma 8 leads to the bound $\left|G^{\prime}\right|=O\left(k^{3}\right)$.

\section{Conclusions and Open Problems}

In this article we have improved the FPT algorithm and kernelization upper bounds for Pathwidth One Vertex Deletion. The kernelization bounds are almost tight compared to lower bounds derived from the general result of Dell and van Melkebeek [6]. However, we do not know of any exact (not FPT) algorithm solving POVD that runs significantly faster than the trivial one (the trivial one, that tries to hit all subgraphs isomorphic to $T_{2}, K_{3}$ and $C_{4}$ runs in $127^{n / 7} \cdot n^{O(1)}=O\left(1.9978^{n}\right)$ time). One approach to obtain a faster exact algorithm would be to develop an FPT algorithm that runs in time $c^{k} n^{O(1)}$ for some constant $c<4$ and use the well-known win-win approach. This, however, seems really nontrivial, as it requires to do something clever with $C_{4}$ subgraphs, instead of simply branching over them.

Acknowledgements This work was partially supported by the Polish Ministry of Science grants N206 491038 and N206 491238.

Open Access This article is distributed under the terms of the Creative Commons Attribution Noncommercial License which permits any noncommercial use, distribution, and reproduction in any medium, provided the original author(s) and source are credited.

\section{References}

1. Àlvarez, C., Serna, M.J.: The proper interval colored graph problem for caterpillar trees (extended abstract). Electron. Notes Discrete Math. 17, 23-28 (2004)

2. Arnborg, S., Proskurowski, A., Seese, D.: Monadic second order logic, tree automata and forbidden minors. In: Börger, E., Büning, H.K., Richter, M.M., Schönfeld, W. (eds.) CSL. Lecture Notes in Computer Science, vol. 533, pp. 1-16. Springer, Berlin (1990)

3. Assmann, S.F., Peck, G.W., Syslo, M.M., Zak, J.: The bandwidth of caterpillars with hairs of length 1 and 2. SIAM J. Algebr. Discrete Methods 2(4), 387-393 (1981)

4. Cao, Y., Chen, J., Liu, Y.: On feedback vertex set new measure and new structures. In: Kaplan, H. (ed.) SWAT. Lecture Notes in Computer Science, vol. 6139, pp. 93-104. Springer, Berlin (2010)

5. Cygan, M., Nederlof, J., Pilipczuk, M., Pilipczuk, M., van Rooij, J.M.M., Wojtaszczyk, J.O.: Solving connectivity problems parameterized by treewidth in single exponential time. In: FOCS (to appear), see also CoRR. arXiv:1103.0534 [cs.Ds] (2011)

6. Dell, H., van Melkebeek, D.: Satisfiability allows no nontrivial sparsification unless the polynomialtime hierarchy collapses. In: Schulman, L.J. (ed.) STOC, pp. 251-260. ACM, New York (2010)

7. Diestel, R.: Graph Theory. Graduate Texts in Mathematics. Springer, Berlin (2006)

8. Downey, R.G., Fellows, M.R.: Parameterized Complexity. Springer, Berlin (1999)

9. Dujmovic, V., Fellows, M.R., Hallett, M.T., Kitching, M., Liotta, G., McCartin, C., Nishimura, N., Ragde, P., Rosamond, F.A., Suderman, M., Whitesides, S., Wood, D.R.: A fixed-parameter approach to 2-layer planarization. Algorithmica 45(2), 159-182 (2006)

10. Fernau, H.: Two-layer planarization: improving on parameterized algorithmics. In: Vojtás, P., Bieliková, M., Charron-Bost, B., Sýkora, O. (eds.) SOFSEM. Lecture Notes in Computer Science, vol. 3381, pp. 137-146. Springer, Berlin (2005) 
11. Flum, J., Grohe, M.: Parameterized Complexity Theory, 1st edn. Texts in Theoretical Computer Science. An EATCS Series. Springer, Secaucus (2006)

12. Lin, M., Lin, Z., Xu, J.: Graph bandwidth of weighted caterpillars. Theor. Comput. Sci. 363(3), 266277 (2006)

13. Lovász, L., Plummer, M.D.: Matching Theory. Annals of Discrete Mathematics. North-Holland, Amsterdam (1986)

14. Mucha, M., Sankowski, P.: Maximum matchings via gaussian elimination. In: FOCS, pp. $248-255$. IEEE Comp. Soc., Los Alamitos (2004)

15. Niedermeier, R.: Invitation to Fixed Parameter Algorithms. Oxford Lecture Series in Mathematics and Its Applications. Oxford University Press, London (2006)

16. Ore, O.: Graphs and matching theorems. Duke Math. J. 22(4), 625-639 (1955)

17. Papadimitriou, C.H.: The NP-completeness of the bandwidth minimization problem. Computing 16(3), 263-270 (1976)

18. Philip, G., Raman, V., Villanger, Y.: A quartic kernel for pathwidth-one vertex deletion. In: Thilikos, D.M. (ed.) WG. Lecture Notes in Computer Science, vol. 6410, pp. 196-207 (2010)

19. Robertson, N., Seymour, P.D.: Graph minors. I. Excluding a forest. J. Comb. Theory, Ser. B 35(1), 39-61 (1983)

20. Robertson, N., Seymour, P.D.: Graph minors. II. Algorithmic aspects of tree-width. J. Algorithms 7(3), 309-322 (1986)

21. Suderman, M.: Layered graph drawing. Ph.D. thesis, School of Computer Science, McGill University Montreál (2005)

22. Thomassé, S.: A $4 k^{2}$ kernel for feedback vertex set. ACM Transactions on Algorithms 6(2), 32:1-32:8 (2010)

23. Uhlmann, J., Weller, M.: Two-layer planarization parameterized by feedback edge set. In: Kratochvíl, J., Li, A., Fiala, J., Kolman, P. (eds.) TAMC. Lecture Notes in Computer Science, vol. 6108, pp. 431-442. Springer, Berlin (2010) 